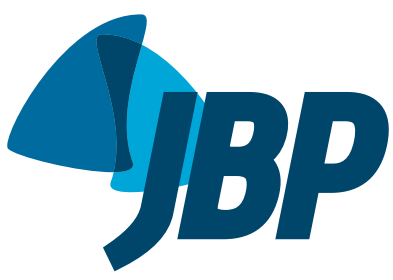

1. Department of Clinical Sciences, Brune University London, United Kingdom.

2. Departamento de Fisioterapia, Faculdade de Medicina, Universidade de São Paulo (SP) Brasil.

a. (iD) http://orcid.org/0000-0002-9500-6223

b. (iD http://orcid.org/0000-0003-3046-3412

c. (iD http://orcid.org/0000-0003-2409-4008

d. (iD http://orcid.org/0000-0002-3588-9060

Submitted: 20 October 2017 Accepted: 17 February 2018

Study carried out in the Department of Clinical Sciences, Brunel University London, London, England, and in the Departamento de Fisioterapia, Faculdade de Medicina, Universidade de São Paulo, São Paulo (SP) Brasil.

\title{
Dysfunctional breathing: what do we know?
}

\author{
Laís Silva Vidotto1,a, Celso Ricardo Fernandes de Carvalho ${ }^{2, b}$, \\ Alex Harvey ${ }^{1, c}$, Mandy Jones ${ }^{1, d}$
}

\begin{abstract}
Dysfunctional breathing (DB) is a respiratory condition characterized by irregular breathing patterns that occur either in the absence of concurrent diseases or secondary to cardiopulmonary diseases. Although the primary symptom is often dyspnea or "air hunger", DB is also associated with nonrespiratory symptoms such as dizziness and palpitations. DB has been identified across all ages. Its prevalence among adults in primary care in the United Kingdom is approximately $9.5 \%$. In addition, among individuals with asthma, a positive diagnosis of DB is found in a third of women and a fifth of men. Although DB has been investigated for decades, it remains poorly understood because of a paucity of high-quality clinical trials and validated outcome measures specific to this population. Accordingly, DB is often underdiagnosed or misdiagnosed, given the similarity of its associated symptoms (dyspnea, tachycardia, and dizziness) to those of other common cardiopulmonary diseases such as COPD and asthma. The high rates of misdiagnosis of DB suggest that health care professionals do not fully understand this condition and may therefore fail to provide patients with an appropriate treatment. Given the multifarious, psychophysiological nature of DB, a holistic, multidimensional assessment would seem the most appropriate way to enhance understanding and diagnostic accuracy. The present narrative review was developed as a means of summarizing the available evidence about DB, as well as improving understanding of the condition by researchers and practitioners
\end{abstract}

Keywords: Hyperventilation; Pulmonary ventilation; Respiratory system; Pulmonary medicine.

\section{INTRODUCTION}

Dysfunctional breathing (DB) is a respiratory condition that is often poorly understood among health care professionals worldwide, leading to its underdiagnosis and misdiagnosis in clinical practice. ${ }^{(1,2)}$ The reasons for its misdiagnosis include a lack of studies investigating its pathophysiology, classification, and symptoms, as well as the similarity between the symptoms of DB and those of common cardiopulmonary diseases, such symptoms including dyspnea, tachycardia, dizziness, and paresthesia. ${ }^{(3)}$ Misunderstandings about the causes, diagnosis, and treatment of DB appear to indicate that health care professionals do not fully understand this condition and may therefore fail to provide patients with an appropriate treatment, which could lead to impaired health-related quality of life (HRQoL). ${ }^{(4)}$ What is known to date is that DB involves different forms of abnormal breathing patterns, ${ }^{(5)}$ and that it affects approximately $9.5 \%$ of all adults in primary care in the United Kingdom. ${ }^{(5)}$ DB has been shown to occur in individuals from 17 to 88 years of age, $(2,6,7)$ being most common in women and in individuals with asthma. ${ }^{(5,8)}$ However, the prevalence of DB can be overestimated or underestimated, given the fact that there is currently no gold-standard tool for diagnosing the condition. Therefore, in this narrative review, we will discuss the definition of $\mathrm{DB}$, as well as the evidence base for its physiological, functional, and psychological characteristics. Our intention is to improve understanding of DB, based on the available scientific evidence, on the part of health care professionals.

\section{DEFINITION AND CLASSIFICATION OF DB}

DB is generally characterized by abnormal breathing patterns $^{(4,9)}$ that occur either in the absence of organic diseases (i.e., due to psychogenic causes such as anxiety) $)^{(10,11)}$ or secondary to cardiopulmonary/neurological diseases (i.e., due to organic/physiological causes such as asthma and heart failure). ${ }^{(5,12)}$ Jones et al. ${ }^{(6)}$ dubbed the former classification "primary $\mathrm{DB}^{\prime}$ and the latter "secondary DB". ${ }^{(6)}$ It is noteworthy that DB is secondary to organic causes when the physiological alterations are insufficient to explain the evident symptoms (e.g., dyspnea) or the blood gas analysis results. ${ }^{(13)}$ Figure 1 shows a representation of "abnormal breathing patterns". It is important to emphasize that those abnormal patterns can appear with greater recruitment of the accessory muscles of respiration or in cases of chronic respiratory alkalosis. ${ }^{(4,14,15)}$

In recent years, researchers have proposed some alternative classifications for different patterns of DB. Barker and Everard ${ }^{(4)}$ reviewed the literature and suggested a new definition of DB: "an alteration in the

Correspondence to:

Laís S. Vidotto. Department of Clinical Sciences, Brunel University London, Kingston Lane, Uxbridge, Middlesex, UB8 3PH, London, UK.

Tel: 440 1895-268-985. Email: lais.vidotto@brunel.ac.uk

Financial support: This study was financed in part by the Coordenação de Aperfeiçoamento de Pessoal de Nível Superior-Brasil (CAPES) - Finance Code 001 
normal biomechanical patterns of breathing that result in intermittent or chronic symptoms which may be respiratory and/or non-respiratory". Among the respiratory symptoms listed by the authors were dyspnea and hyperventilation, whereas their list of nonrespiratory symptoms included dizziness and tachycardia. The authors also highlighted the fact that pattern disordered breathing is the main component of any form of DB. Table 1 shows the classifications of DB proposed by various authors. The evident differences across studies regarding the classification of DB and the terminology employed in describing it underscore the need to move from an incipient stage (of discovery) to a more developed phase (of diagnosis and treatment).

The most well-known form of DB is hyperventilation syndrome (HVS), which is defined as acute or chronic hyperventilation (increased minute volume) at rest or during exercise/stress. ${ }^{(16,17)}$ As a form of DB, HVS may be due to organic/physiological conditions but is mainly caused by psychological/behavioral factors (e.g., anxiety, depression, perfectionism, and feelings of inferiority). ${ }^{(16,18)}$ For many years, it was thought that HVS was always accompanied by hypocapnia, which can trigger nonrespiratory symptoms such as dizziness, palpitations, numbness, and a tingling sensation. ${ }^{(19)}$ However, recent studies indicate that such symptoms could be triggered by other, as yet unknown, factors, given that respiratory alkalosis is not always present in HVS. $(4,13,20)$

There is another form of DB, known as idiopathic hyperventilation $(\mathrm{IH})$, in which patients have chronic asymptomatic hyperventilation and respiratory alkalosis, that cannot be attributed to an underlying disease. ${ }^{(18)}$ Jack et al. ${ }^{(21)}$ showed that IH is mainly caused by psychological factors, becoming a conditioned response. In a subsequent study, Jack et al. ${ }^{(18)}$ hypothesized that $\mathrm{IH}$ is caused by increased respiratory responsiveness to carbon dioxide levels, to hypoxia, or to an increase

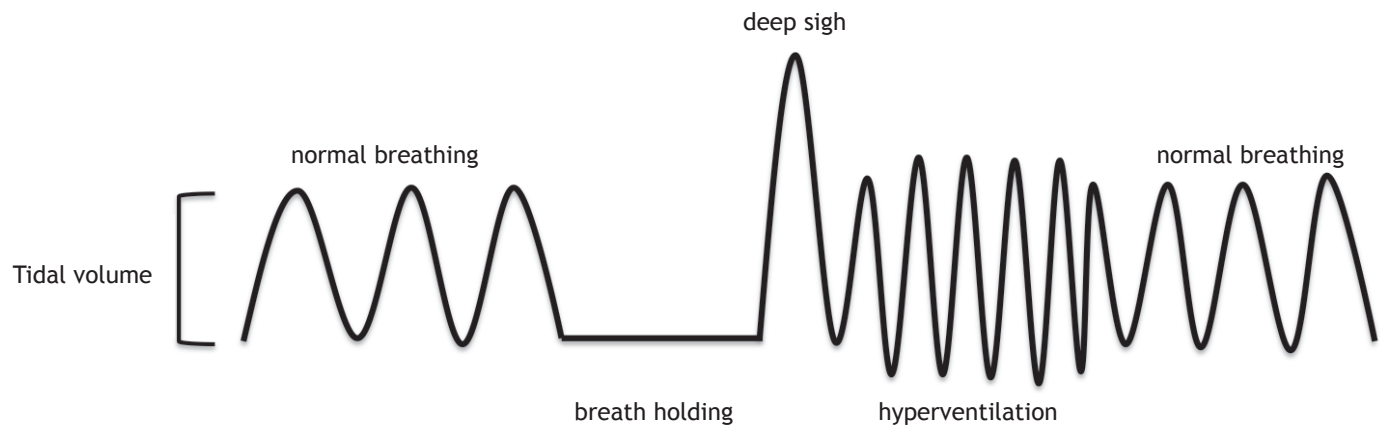

Figure 1. Representative figure showing the "normal" breathing patterns and the "abnormal" breathing patterns presented by individuals with dysfunctional breathing. Normal breathing is the standard tidal volume and respiratory rate of an individual; breath holding is when a breath is held for a period of time; a deep sigh is a deep inspiration that happens at any time during breathing; and hyperventilation is an increase in the respiratory rate, tidal volume, or both. Note: The order in which abnormal breathing patterns appear may vary.

Table 1. Recent classifications of dysfunctional breathing suggested by different authors.

Boulding et al. ${ }^{(9)}$

Hyperventilation syndrome

Periodic deep sighing

Thoracic dominant breathing

Forced abdominal expiration

Thoracoabdominal asynchrony

Barker and Everard ${ }^{(4)}$

Thoracic DB

Extrathoracic DB

Functional DB (a subdivision of thoracic and extrathoracic DB)

Structural DB (a subdivision of thoracic and extrathoracic DB)
Related to respiratory alkalosis or independent of hypocapnia Usually associated with an irregular breathing pattern Can manifest more often in somatic diseases

Evident when there is inappropriate and excessive abdominal muscle contraction during expiration

Characterized by a delay between intercostal and abdominal contraction, causing ineffective respiratory mechanics

Significant changes in the breathing pattern that may or may not be directly linked to hyperventilation

Upper airway impairment manifested in combination with breathing pattern disorders (e.g., vocal cord dysfunction)

No structural or functional alterations directly associated with the symptoms of DB (e.g., phrenic nerve palsy, myopathy, and diaphragmatic eventration)

Primarily associated with anatomical or neurological alterations (e.g., subglottic stenosis and unilateral cord palsy) 
in the metabolic rate, although that hypothesis has not been tested.

\section{DIAGNOSIS OF DB}

\section{Nijmegen Questionnaire}

Currently, the Nijmegen Questionnaire (NQ) is the most widely used instrument for identifying individuals with DB. ${ }^{(22,23)}$ The NQ was developed by van Dixhoorn and Duivenvoorden in 1985. ${ }^{(23)}$ Although the authors did not specify whether the participants had primary or secondary HVS, they mentioned the exclusion of participants with somatic disorders, which would suggest the exclusion of those with primary DB. Nevertheless, they included no information on whether any of the participants had a history of asthma or other health conditions.

The NQ consists of 16 questions related to complaints, and the frequency of symptoms can be indicated on a scale ranging from 1 (never) to 5 (very often). It has a sensitivity and specificity of $91 \%$ and $95 \%$, respectively, in relation to the clinical diagnosis of HVS. The questions are primarily related to physiological factors (e.g., cardiovascular and respiratory symptoms) and psychological factors (e.g., tension). However, it is noteworthy that this instrument was developed as a symptom-based questionnaire to screen for HVS. ${ }^{(23)}$ In fact, the NQ has been validated only for use in patients with HVS secondary to asthma (i.e., not for use in those with other forms of DB). ${ }^{(24)}$ In addition, the NQ has yet to be translated to Portuguese or validated for use in Brazil. In a recent article, one of the creators of the NQ stated that it has been used incorrectly worldwide, ${ }^{(25)}$ stating that it should be used only to identify abnormal symptoms related to HVS and not as the gold-standard test to diagnose DB per se, because it was not created for that purpose. However, given that HVS was the first form of DB to be investigated and is still the most well understood form of the condition, several tools, other than the NQ, have been developed to facilitate the identification of hyperventilation (as described below).

\section{The hyperventilation provocation test to identify HVS}

The hyperventilation provocation test (HVPT) was once considered to be the cornerstone of the diagnosis of HVS. (26) The HVPT requires patients to breathe as deeply and quickly as possible for a certain length of time (typically 2 or $3 \mathrm{~min}$ ). To determine whether patients have HVS, the authors who developed the HVPT suggested that clinicians check patient reports of symptoms and experiences during the test. If a patient reports symptoms and sensations that are similar to those experienced regularly in their daily lives, the presence of HVS can be confirmed. (27) However, a number of researchers have begun to question whether the HVPT is a valid tool to detect HVS, because it does not differentiate between symptoms caused by hypocapnia and those caused by task-related anxiety or mechanical discomfort. ${ }^{(28)}$ In such instances, the HVPT is highly likely to overestimate the prevalence of HVS. ${ }^{(13,28)}$ In addition, a delay for blood $\mathrm{CO}_{2}$ levels to return to baseline values after an HVPT was once believed to be diagnostic of HVS. (27) However, it was later shown that the length of the delay in $\mathrm{CO}_{2}$ recovery does not differ between patients with HVS and healthy individuals. ${ }^{(28)}$

\section{Cardiopulmonary exercise testing to identify HVS}

Another means of facilitating the diagnosis of HVS is cardiopulmonary exercise testing. ${ }^{(18,20)}$ Such testing has been recommended when patients do not experience HVS-related symptoms at rest but health care professionals suspect that, based on patient reports, these sensations are likely to occur during movement. $(18,20)$ That assumption is predicated on compelling evidence indicating that, on incremental cycle ergometer tests, individuals with chronic IH show lower end-tidal $\mathrm{CO}_{2}$ tension, higher minute volume, slower recovery, and a higher degree of dyspnea (disproportionate to the increase in minute volume), as well as supporting lower workloads, than do healthy individuals. ${ }^{(18,20,21)}$ Although the use of cardiopulmonary exercise testing might also facilitate the exclusion of other causes of dyspnea, ${ }^{(29)}$ the protocol for the identification of HVS during cardiopulmonary exercise testing has yet to be standardized. Therefore, exercise tests appear to be complementary tools that can facilitate the detection of HVS in more complex situations, in which patients do not show any of the aforementioned symptoms at rest.

\section{PHYSIOLOGICAL CHARACTERISTICS OF DB}

\section{Pulmonary function}

Although pulmonary function test parameters, such as $\mathrm{FEV}_{1}$ and FVC, are widely used to classify respiratory diseases, they do not seem to distinguish patients with DB. Data in the literature describing pulmonary function in DB are extremely divergent. ${ }^{(30,31)}$ There is compelling evidence that individuals with primary DB have normal lung function, ${ }^{(1,2,17,30)}$ whereas other studies have shown that $\mathrm{FEV}_{1}$ or FVC are $10-15 \%$ below the predicted values in such individuals. ${ }^{(17,31)}$ In addition, Agache et al. ${ }^{(32)}$ showed that $\mathrm{FEV}_{1}$ declined faster over a 12-month period in patients with asthma-induced DB (secondary DB) than in those with asthma alone. Furthermore, Jones et al. ${ }^{(6)}$ reported that a 26 -week breathing retraining program improved $\mathrm{FEV}_{1}$ in patients with primary DB. These findings can be explained by the fact that some patients with DB hyperventilate, which causes hypocapnia, accompanied by reduced airway caliber and increased airway resistance. ${ }^{(33,34)}$ It is well established that, when trial data are reported, pulmonary function test results should be provided as raw data (in $\mathrm{L}$ or $\mathrm{mL}$ ) and as percentages of the predicted values, with the use of reference equations that are specific to the studied population. ${ }^{(35)}$ However, most 
authors investigating DB (either primary or secondary) have evaluated changes in lung function by analyzing only raw data and not percentage data. $(1,2,17,30-32)$ Others have presented the data as percentages of the predicted values without indicating the reference equations used in their calculation. ${ }^{(17,30-32)}$

Courtney et al. ${ }^{(30)}$ used spirometry to assess pulmonary function in a mixed group of healthy individuals and individuals with mild medical conditions (including suspected DB). Following the pulmonary function tests, participants were divided in two groups, on the basis of the results of those tests: normal and abnormal. The authors found that, in comparison with the participants who showed normal lung function, those with abnormal lung function had lower blood $\mathrm{CO}_{2}$ levels, shorter breath-holding times, and lower oxygen saturation, as well as scoring higher (i.e., worse) on the Self-Evaluation of Breathing Questionnaire. ${ }^{(30)}$ These results suggest that there is a group of patients with $D B$ and chronic hyperventilation (and therefore hypocapnia) who have abnormal lung function. Therefore, it seems that any conclusion regarding pulmonary function in DB can be considered an attempt to simplify the multifaceted phenomena seen in individuals with DB.

It is important to emphasize that researchers and health care professionals should not expect to find substantially abnormal spirometry results in individuals with primary $D B$, because it is not considered a respiratory disorder. Despite the fact that hypocapnia has an influence on airway caliber (i.e., constricts the airways), ${ }^{(33,34)}$ not all patients with DB exhibit hyperventilation. Therefore, the reported degree of pulmonary function impairment varies widely across studies of DB, which justifies attempts to investigate this parameter in greater depth.

\section{Respiratory muscle function}

A number of studies have demonstrated abnormal function of the respiratory muscles (e.g., hyperventilation), ${ }^{(1,11,36)}$ with or without greater recruitment of the accessory muscles of respiration, in patients with primary DB. ${ }^{(6,31,37)}$ In healthy individuals, common situations such as emotional stress cause predominantly thoracic breathing, muscle tension, and increased respiratory muscle effort. ${ }^{(38)}$ When such symptoms are persistent and have no physiological cause, they are suggestive of DB. ${ }^{(36)}$ The accessory muscles of respiration also provide postural control and facilitate breathing during stressful situations, exercise, or threatening situations. ${ }^{(39,40)}$ Emotional stress and anxiety cause abdominal muscle tension, which inhibits diaphragmatic movement. That can induce thoracic breathing and greater recruitment of the accessory muscles of respiration, ${ }^{(6,41)}$ resulting in dyspnea, ${ }^{(42,43)}$ increased work of breathing, ${ }^{(11)}$ and respiratory muscle fatigue. ${ }^{(44)}$

In various respiratory conditions (e.g., COPD and asthma), the degree to which the accessory muscles are recruited indicates the severity of the disease. ${ }^{(45,46)}$ The accessory muscle recruitment caused by psychological conditions frequently associated with DB could result in general muscle tenderness, especially in the upper chest, shoulders, and neck. ${ }^{(47)}$ In addition, some patients with DB exhibit audible, forced expiration, ${ }^{(47)}$ which could be indicative of inappropriate expiratory muscle activation at rest. Many authors have speculated on whether or not respiratory muscle function (RMF) is impaired in patients with DB. ${ }^{(48)}$ Therefore, there seems to be insufficient evidence in the literature to support a positive or negative theoretical position regarding respiratory muscle dysfunction as a feature of $D B$.

The existing literature indicates that RMF in DB has primarily been evaluated by measuring the breathing pattern at rest as a means of identifying thoracic/ paradoxical breathing, through the use of techniques such as manual assessment of respiratory motion. ${ }^{(36,37)}$ However, measurements of breathing pattern performed at rest provide no objective information regarding the contractile properties of the respiratory muscles. The manual assessment of respiratory motion technique has been shown to have better inter-rater reliability and validity than does respiratory inductance plethysmography ${ }^{(49)}$ and is considered a valuable tool in the clinical field, where practitioners have limited access to laboratory equipment. However, more robust physiological measures of RMF, such as muscle strength, endurance and mechanical power, should also be considered in this population. ${ }^{(50)}$

According to Agache et al.,(32) the treatment for DB frequently includes strengthening the diaphragm muscle. However, in the present review, we identified no studies applying respiratory muscle training in DB. In addition, there is no evidence to support the idea that RMF is impaired in patients with DB; nor is there any evidence that inspiratory muscle training has beneficial effects in this population. It is possible that patients with primary DB develop dynamic hyperinflation during hyperventilation, which would impair RMF by shortening and weakening the muscles. However, that theory remains untested. Given the paucity and inconclusive nature of the data currently available ${ }^{(32,50)}$ we highly recommended the direct assessment of RMF.

\section{Cardiopulmonary interactions}

Respiration exerts a potent influence on the autonomic system. ${ }^{(51)}$ Slow, controlled breathing ( $\approx 6$ breaths/ $\mathrm{min}$ ) increases the amplitude of respiratory sinus arrhythmia (RSA), which is a time-domain index of heart rate variability (HRV). ${ }^{(52)}$ In addition to being a robust index of HRV, RSA is a complex physiological phenomenon; the function of which has yet to be fully understood. The amplitude of RSA (and therefore HRV) decreases during tachypneic or erratic breathing patterns. Patients with COPD show lower HRV, ${ }^{(53)}$ which could be attributed to their abnormal breathing pattern. Furthermore, low HRV has been associated with negative prognostic indicators in a number of patient populations (e.g., patients with COPD and patients on hemodialysis). ${ }^{(54,55)}$ Accordingly, given the well-established synchrony between the respiratory and 
cardiovascular systems, ${ }^{(51)}$ it is likely that, in patients with DB, cardiovascular activity is atypical (e.g., an altered pattern of arterial blood pressure and HRV). Therefore, if a patient with DB is taught to breathe in a controlled manner, at a lower respiratory rate, sympathetic activity can be attenuated, ${ }^{(52)}$ a physiological mechanism that could optimize/facilitate gas exchange and mitigate the symptoms of DB.

Courtney et al. ${ }^{(56)}$ detected a significant relationship between a predominantly thoracic breathing pattern and HRV indices that reflect cardiopulmonary efficiency in patients with DB. In the past, it has been argued that hyperventilation is a major cause of cardiac symptoms, due to the cerebral and peripheral vasoconstriction occurring secondary to hypocapnia. ${ }^{(26)}$ It has been suggested that the underlying mechanism is hyperventilation and the consequent hypocapnia, which suppresses sympathetic and parasympathetic activities, the greatest decrease being observed in the parasympathetic activity in the heart. $(4,57)$ Hyperventilation also induces a reduction in cerebral blood flow as a result of hypocapnia; this mechanism is likely to account for the symptoms commonly reported in patients with $\mathrm{DB}$, such as dizziness and unsteadiness. ${ }^{(47)}$ Although there are psychophysiological reactions associated with $\mathrm{DB}$, it remains unclear whether episodes of irregular breathing pattern have an influence (direct or indirect) on the autonomic nervous system.

\section{Gas exchange and ventilation}

It has been suggested that patients with DB expend more energy at rest because of their irregular breathing pattern, (11) which could be explained by anxiety-related factors and increased respiratory muscle work. However, Malmberg et al. ${ }^{(58)}$ showed that, when the participants were in the supine position, gas exchange variables were similar between the patients with HVS and healthy individuals. Nevertheless, when the participants were in a standing position, the patients with HVS had lower levels of expired $\mathrm{CO}_{2}$ and higher minute ventilation $\left(V_{E}\right)$, as well as higher ventilatory equivalents for oxygen $\left(\mathrm{V}_{\mathrm{E}}\right.$ /oxygen uptake), carbon dioxide $\left(\mathrm{V}_{\mathrm{E}} /\right.$ carbon dioxide production), oxygen (minute volume/ oxygen uptake), and $\mathrm{CO}_{2}$ (minute volume/carbon dioxide production), than did the healthy individuals. In addition, Jack et al. ${ }^{(18)}$ reported that patients with IH (i.e., hyperventilation associated with low carbon dioxide production, unrelated to other diseases and not associated with fear) exhibit sustained hyperventilation, higher dyspnea, and lower ventilatory responsiveness to hypoxia during an incremental cycle ergometer test, in comparison with healthy individuals. However, the authors found that those same patients showed variable energy expenditure levels, as well as normal responses to hypoxia and normocapnia at a higher $\mathrm{CO}_{2}$ level $(40 \mathrm{mmHg})$. Therefore, the energy expenditure and ventilation levels of patients with DB have hitherto been under-researched. Those components are of great relevance to furthering understanding of this respiratory condition and should not be overlooked during the collection, analysis, and interpretation of data.

\section{FUNCTIONAL CHARACTERISTICS OF DB}

\section{Functional assessments}

In patients with $\mathrm{DB}$, functional assessments (e.g., breath-holding tests and visual evaluation of breathing patterns) are commonly used in order to complement the identification of this respiratory condition. (17) However, there is little evidence of any strong correlations between these functional outcomes and the underlying physiological parameters. ${ }^{(17,56)}$ There is also insufficient evidence to support direct correlations between functional outcomes and the score on the NQ. ${ }^{(24,31,36)}$ In addition, there are no validated tools specifically designed to assess individuals with DB. ${ }^{(31)}$

In 2011, Courtney et al. ${ }^{(17)}$ investigated a group of individuals who had concerns about their breathing but had not received a diagnosis of primary or secondary DB. The authors identified weak to non-existent correlations among biochemical indices (e.g., lower end-tidal $\mathrm{CO}_{2}$ tension), breath-holding time, and symptom-related questionnaire scores. In a more recent study, involving patients with primary HVS, quality of life (QoL) measurements (domains of the Medical Outcomes Study 36-item Short-Form Health Survey) were found to correlate moderately with $\mathrm{NQ}$ scores, peak respiratory rate, and end-tidal $\mathrm{CO}_{2}$ tension during cardiopulmonary exercise testing. ${ }^{(59)}$ As was evident in the aforementioned studies, there is as yet insufficient evidence of correlations between functional parameters and DB-related symptoms and there is a need for further investigation. Future studies should also aim to recruit a larger number of patients with DB in order investigate the influence of DB on function-related tasks, such as the breath-holding test.

\section{Exercise capacity}

Exercise capacity is a parameter commonly affected by respiratory diseases such as COPD and cystic fibrosis. ${ }^{(60,61)}$ However, there have been few studies employing exercise capacity tests and performing physiological/psychological assessments during exercise in patients with $D B$, and the few that have been conducted have produced controversial results. Such studies typically recruit patients with HVS or secondary DB, as described below. Some studies have also employed progressive exercise tests to confirm the diagnosis of HVS, as identified by the presence of hyperventilation during exercise and at rest, in patients with asthma. ${ }^{(20)}$

Warburton and Jack ${ }^{(20)}$ suggested that cardiopulmonary exercise testing is the most sensitive tool to confirm the diagnosis of HVS, which can be established when patients hyperventilate during exercise performed during exercise activities, such as running and cycling. However, it remains unknown whether exercise performance outcomes or variables collected during exercise are somehow useful in the assessment of 
other forms of DB. Chenivesse et al.(59) employed maximal cardiopulmonary exercise testing on a cycle ergometer in patients with HVS and recorded a range of respiratory variables. ${ }^{(59)}$ The authors found that the patients with HVS showed exercise-related impairments (e.g., aerobic capacity below $84 \%$ of the predicted value) and hyperventilated not only at rest but also (and perhaps even more) during exercise. We find it interesting that most of the patients with HVS in that study showed normal changes in the breathing pattern and a normal increase in respiratory rate, as well as normal cardiovascular responses, during maximal cardiopulmonary exercise testing. However, it is noteworthy that the authors compared their results with those of other studies investigating healthy participants; in other words, there was no direct comparison between patients with HVS and healthy individuals in their study.

Howell(13) suggested that symptoms of HVS occur predominately at rest but can occur during exercise, proposing that such symptoms are not directly associated with the intensity of the exercise performed. ${ }^{(13)}$ Jack et al. ${ }^{(21)}$ systematically investigated that proposition and found it to hold true. In the latter study, a group of patients were observed to hyperventilate prior to or at the onset of the exercise test, a psychological condition dubbed anticipatory anxiety. ${ }^{(62)}$ In such instances, patients tend to hyperventilate when facing a challenging situation. It is noteworthy that there is also a subcategory of individuals with chronic hyperventilation who show appropriate changes in $\mathrm{CO}_{2}$ from rest to exercise or from rest to sleeping and possess exercise capacity similar to that of healthy participants. Therefore, Jack et al. ${ }^{(21)}$ suggested that psychological variables such as anxiety-related factors should be assessed in this population, not only at rest but also in exercise-related situations. ${ }^{(21)}$ More recently, Courtney et al. ${ }^{(17)}$ argued that, when measurements of $\mathrm{CO}_{2}$ at rest are not suggestive of $\mathrm{DB}, \mathrm{CO}_{2}$ should be measured during an exercise test, in a challenging situation (e.g., retrieving an old memory), or both. Similarly, Hagman et al. ${ }^{(1)}$ identified breathing difficulties during exercise in patients with $\mathrm{DB}$ and in patients with asthma. In a subsequent study, the same group of authors found that a one- to three-month breathing retraining program ameliorated breathing difficulties during exercise. (2) Notably, that improvement was maintained over a 5-year follow-up period. There is a need for systematic studies assessing exercise capacity, as well as physiological and psychological changes during exercise, in patients with primary DB. Given that HVS is a subcategory of DB and appears to have a negative effect during exercise, it is extremely relevant that the results of exercise capacity tests are taken into consideration when patients are suspected of having DB. Evaluation of individuals during exercise sessions or other stressful situations to diagnose DB could provide health care professionals with a broader, richer, and more nuanced perspective of the nature of the condition, as well as facilitating/expediting its diagnosis and treatment.

\section{PSYCHOSOCIAL CHARACTERISTICS OF DB}

\section{Anxiety and depression}

Although respiratory and cardiovascular parameters undergo significant changes in emotionally challenging situations (i.e., the "fight-or-flight response" occurs) in all individuals, patients with DB commonly experience abnormal psychophysiological responses when facing similar scenarios. ${ }^{(9)}$ DB induces significant changes in the respiratory rate, breath-holding time, and depth of breathing that are primarily mediated by current or previous traumatic experiences or psychological conditions (e.g., anxiety). These symptoms may also occur chronically, which leads to deterioration of the QoL of the individual. ${ }^{(1,63)}$ Accordingly, a strong correlation between psychological condition and changes in normal breathing has been identified in different contexts (e.g. during musical performances) in healthy individuals. ${ }^{(64)}$ Anxiety and depression are common among patients with HVS. ${ }^{(10,21,47,65)}$ In fact, Hagman et al. ${ }^{(1)}$ showed that the addition of DB to asthma (secondary DB) is associated with higher levels of anxiety and depression. However, the incidence of anxiety/depression in DB and whether such disorders are essential factors in the identification of $D B$ remain unclear and require further research.

\section{QoL}

Some authors have suggested that symptoms of DB secondary to asthma can influence HRQoL, as determined by the Asthma Quality of Life Questionnaire and the Medical Outcomes Study 36-item Short-Form Health Survey, two well-established tools for the assessment of HRQoL. ${ }^{(66,67)}$ Hagman et al. ${ }^{(1,2)}$ and Chenivesse et al. ${ }^{(59)}$ assessed QoL in individuals with primary HVS and found that HRQoL was lower in those individuals than in groups of healthy participants. Hagman et al. ${ }^{(2)}$ also proposed that breathing retraining had a positive effect on QoL even at the end of a 5-year follow-up period. However, a Cochrane systematic review investigating treatment for DB identified only one randomized controlled trial (RCT) in this field of research, and that trial did not include an assessment of QoL. ${ }^{(15)}$ Given that DB may be associated with impaired QoL, it is crucial to gain greater insight and understanding of the impact that DB has on this parameter. Therefore, we believe that further research should be conducted in order to elucidate the psychophysiological and behavioral mechanisms that underlie DB. By identifying such triggers, clinicians are more likely to provide patients with an appropriate and effective treatment that will have various positive consequences on QoL.

\section{TREATMENT OF DB}

There are few data in the literature regarding treatment for patients with DB. That paucity can be explained by differences across studies in terms of the recruitment methods employed, which is understandable because that there is no standardized diagnostic tool available for DB. Therefore, it is extremely important to 
be cautious when considering these results in research or clinical practice.

In 2013, Jones et al.(15) published a systematic review looking at RCTs in which DB was treated with breathing exercises and the effects that those exercises had on patient QoL were evaluated. The authors identified only one such RCT, conducted in the Netherlands, ${ }^{(68)}$ in which 41 patients with HVS were evaluated. In that study, there were two intervention groups (relaxation therapy only and relaxation therapy plus breathing exercises) and a control group. The relaxation therapy was based on a combination of yoga techniques and the method devised by Jacobson. ${ }^{\left({ }^{69}\right)}$ The breathing exercises focused on reducing the respiratory rate and encouraging diaphragmatic breathing. Both intervention groups were treated in ten $60-\mathrm{min}$ sessions. The study had two main outcome measures: the number and intensity of hyperventilation attacks; and the symptoms experienced. However, the authors did not specify how those parameters were assessed. The results show that there was a significant reduction in number and intensity of hyperventilation attacks in both intervention groups in comparison with the control group, the patients in the relaxation therapy plus breathing exercises group improving more than did those in the relaxation therapy-only group.

Apart from the abovementioned RCT, the main therapeutic approach utilized for patients with $D B$ is breathing retraining. ${ }^{(2,6)}$ In 2003, Thomas et al. ${ }^{(66)}$ found that patients with DB secondary to asthma showed significant symptom improvement after breathing retraining. In 2011, Hagman et al.(2) concluded that patients with $D B$ benefit from breathing retraining, even at the end of a 5-year follow-up period. In 2015, Jones et al. ${ }^{(6)}$ found that manual therapy does not add benefit to breathing retraining in the same population. However, the differences among recruitment methods, as well as among breathing retraining programs, and the lack of descriptions of protocols show the need for further clinical trials in this field of research.

\section{FINAL CONSIDERATIONS}

Due to the multifaceted, complex nature of $D B$, a holistic multidimensional assessment is required for the accurate diagnosis of this respiratory condition. That being the case, three key domains-biochemical aspects, biomechanical aspects, and respiratory symptoms-require further investigation in order to improve our understanding of DB. ${ }^{(17,25)}$ In addition, based on our critical analysis of the existing literature, we believe that psychological, social, and physiological domains also have a significant impact on the pathology and severity of DB (Figure 2). Finally, treatment for patients with DB needs to be better investigated, not only because of the lack of a diagnostic tool that would enable consistent recruitment of participants but also because of the scarcity of RCTs testing well-defined protocols for this patient group.

The lack of studies investigating the respiratory symptoms of $D B$, as well as its biochemical, biomechanical, psychological, social, and physiological aspects, perpetuates poor understanding and often results in delayed diagnosis of the condition. Therefore, the development of a multidimensional assessment tool specifically for DB, as well as a set of standardized clinical outcome measurements to identify and monitor treatment efficacy, are essential prerequisites to improving the provision of effective health care services for individuals with $\mathrm{DB}$.

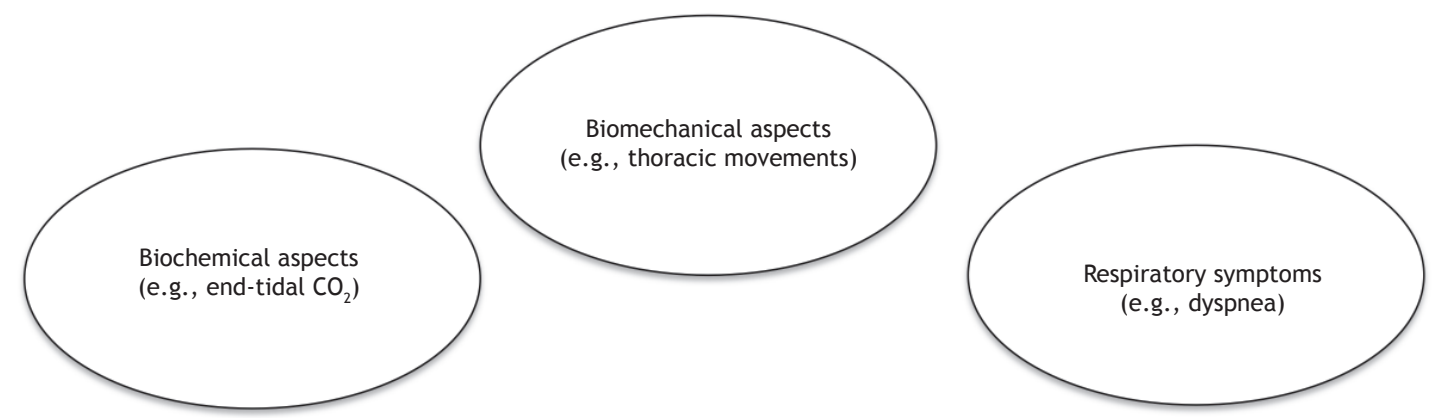

Multidimensional diagnosis of dysfunctional breathing

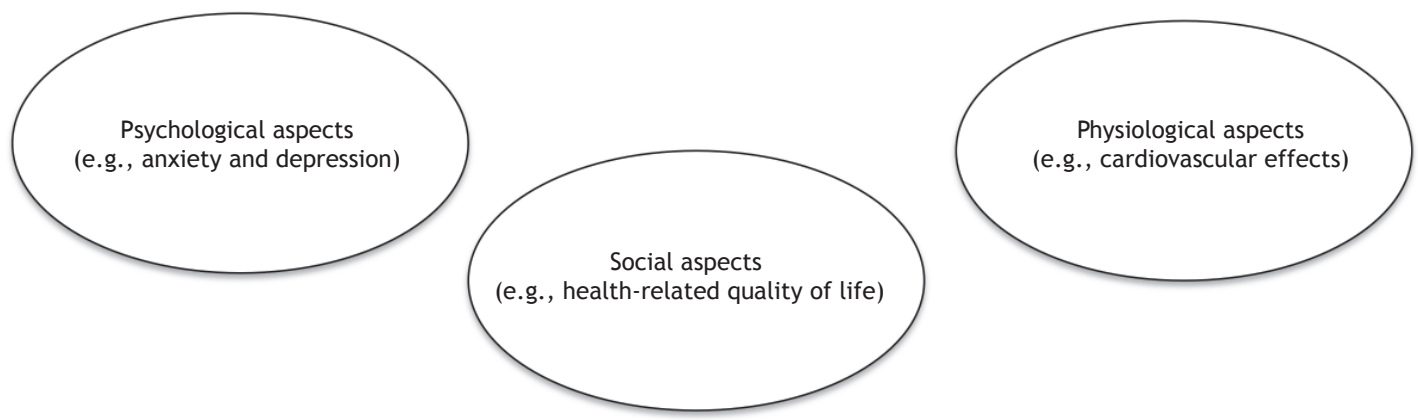

Figure 2. Representative diagram of the proposed aspects involved in the multidimensional diagnosis of dysfunctional breathing. 


\section{REFERENCES}

1. Hagman $\mathrm{C}$, Janson $\mathrm{C}$, Emtner M. A comparison between patients with dysfunctional breathing and patients with asthma. Clin Respir J 2008;2(2):86-91. https://doi.org/10.1111/j.1752-699X.2007.00036.x

2. Hagman $C$, Janson $C$, Emtner M. Breathing retraining - a five-year follow-up of patients with dysfunctional breathing. Respir Med. 2011;105(8):1153-9. https://doi.org/10.1016/J.rmed.2011.03.006

3. Barker NJ, Jones M, O'Connell NE, Everard ML. Breathing exercises for dysfunctional breathing/hyperventilation syndrome in children. Cochrane Database Syst Rev. 2013;12(12):CD010376. https://doi org/10.1002/14651858.CD010376

4. Barker N, Everard ML. Getting to grips with "dysfunctional breathing." Paediatr Respir Rev. 2015;16(1):53-61. https://doi. org/10.1016/j.prrv.2014.10.001

5. Thomas M, McKinley RK, Freeman E, Foy C, Price D. The prevalence of dysfunctional breathing in adults in the community with and without asthma. Prim Care Respir J. 2005;14(2):78-82. https://doi. org/10.1016/j.pcrj.2004.10.007

6. Jones M, Troup F, Nugus J, Roughton M, Hodson M, Rayner C, et al. Does manual therapy provide additional benefit to breathing retraining in the management of dysfunctional breathing? A randomised controlled trial. Disabil Rehabil. 2015;37(9):763-70. https://doi.org/10.3109/09638288.2014.941020

7. De Groot EP, Duiverman EJ, Brand PL. Dysfunctional breathing in children with asthma: a rare but relevant comorbidity. Eur Respir $\mathrm{J}$ 2013:41(5):1068-73. https://doi.org/10.1183/09031936.00130212

8. Thomas M, McKinley RK, Freeman E, Foy C. Prevalence of dysfunctional breathing in patients treated for asthma in primary care: cross sectional survey. BMJ. 2001;322(7294):1098-100 https://doi.org/10.1136/bmj.322.7294.1098

9. Boulding R, Stacey R, Niven R, Fowler SJ. Dysfunctional breathing a review of the literature and proposal for classification. Eur Respir $\mathrm{J}$. 2016;25(141):287-94. https://doi.org/10.1183/16000617.0088-2015

10. Han JN, Stegen K, De Valck C, Clément J, Van de Woestijne KP. Influence of breathing therapy of complaints, anxiety, and breathing pattern with hyperventilation syndrome and anxiety disorders. $J$ Psychosom Res. 1996:41(5):481-93. https://doi.org/10.1016/S0022 3999(96)00220-6

11. Gilbert C. Emotional sources of dysfunctional breathing. J Bodyw Mov Ther. 1998;2(4):224-30. https://doi.org/10.1016/S13608592(98)80019-3

12. DeGuire S, Gevirtz R, Hawkinson D, Dixon K. Breathing retraining a three-year follow-up study of treatment for hyperventilation syndrome and associated functional cardiac symptoms. Biofeedback Self Regul. 1996;21(2):191-8. https://doi.org/10.1007/BF02284695

13. Howell JB. The hyperventilation syndrome: a syndrome unde threat? Thorax. 1997;52 Suppl 3:S30-4. https://doi.org/10.1136/ thx.52.2008.S30

14. Bott J, Blumenthal S, Buxton M, Ellum S, Falconer C, Garrod R, et al Guidelines for the physiotherapy management of the adult, medical spontaneously breathing patient. Thorax. 2009;64 Suppl 1:i1-51. https://doi.org/10.1136/thx.2008.110726

15. Jones M, Harvey A, Marston L, O'Connell NE. Breathing exercises for dysfunctional breathing/hyperventilation syndrome in adults Cochrane Database Syst Rev. 2013;(5):CD009041 https://doi org/10.1002/14651858.CD009041.pub2

16. Gardner WN. The pathophysiology of hyperventilation disorders Chest. 1996;109(2):516-34. https://doi.org/10.1378/chest.109.2.516

17. Courtney R, Greenwood KM, Cohen M. Relationships between measures of dysfunctional breathing in a population with concerns about their breathing. J Bodyw Mov Ther. 2011;15(1):24-34. https:// doi.org/10.1016/j.jbmt.2010.06.004

18. Jack S, Rossiter HB, Pearson MG, Ward SA, Warburton CJ, Whipp BJ. Ventilatory responses to inhaled carbon dioxide, hypoxia, and exercise in idiopathic hyperventilation. Am J Respir Crit Care Med. 2004;170(2):118-25. https://doi.org/10.1164/rccm.200207-7200C

19. Kerr WJ, Gliebe PA, Dalton JW. Physical Phenomena Associated with Anxiety States: The Hyperventilation Syndrome. Cal West Med. 1938;48(1):12-6

20. WarburtonCJ, JackS. Canyou diagnosehyperventilation? ChronRespi Dis. 2006;3(3):113-5. https://doi.org/10.1191/1479972306cd116ed

21. Jack S, Rossiter HB, Warburton CJ, Whipp BJ. Behavioral influences and physiological indices of ventilatory control in subjects with idiopathic hyperventilation. Behav Modif. 2003:27(5):637-52. https:// doi.org/10.1177/0145445503256318
22. Vansteenkiste J, Rochette F, Demedts M. Diagnostic tests of hyperventilation syndrome. Eur Respir J. 1991;4:393-9.

23. van Dixhoorn J, Duivenvoorden HJ. Efficacy of Nijmegen Questionnaire in recognition of the hyperventilation syndrome. J Psychosom Res. 1985;29(2):199-206. https://doi.org/10.1016/00223999(85)90042-X

24. Grammatopoulou EP, Skordilis EK, Georgoudis G, Haniotou A Evangelodimou A, Fildissis G, et al. Hyperventilation in asthma: a validation study of the Nijmegen Questionnaire--NQ. J Asthma. 2014;51(8):839-46. https://doi.org/10.3109/02770903.2014.922190

25. van Dixhoorn J, Folgering $H$. The Nijmegen Questionnaire and dysfunctional breathing. Eur Respir J. 2015;1(1). pii: 00001-2015.

26. Hegel MT, Abel GG, Etscheidt M, Cohen-Cole S, Wilmer Cl. Behaviora treatment of angina-like chest pain in patients with hyperventilation syndrome. J Behav Ther Exp Psychiatry. 1989;20(1):31-9. https://doi org/10.1016/0005-7916(89)90005-0

27. Grossman P, de Swart JC. Diagnosis of hyperventilation syndrome on the basis of reported complaints. J Psychosom Res. 1984;28(2):97 104. https://doi.org/10.1016/0022-3999(84)90001-1

28. Hornsveld HK, Garssen B, Dop MJ, van Spiegel PI, de Haes JC Double-blind placebo-controlled study of the hyperventilation provocation test and the validity of the hyperventilation syndrome. Lancet. 1996;348(9021):154-8. https://doi.org/10.1016/S0140 6736(96)02024-7

29. American Thoracic Society; American College of Chest Physicians ATS/ACCP Statement on cardiopulmonary exercise testing. Am J Respir Crit Care Med. 2003;167(2):211-77. https://doi.org/10.1164/ rccm.167.2.211

30. Courtney $R$, Cohen M. Investigating the claims of Konstantin Buteyko, M.D., Ph.D.: The relationship of breath holding time to end tidal $\mathrm{CO} 2$ and other proposed measures of dysfunctiona breathing. J Altern Complement Med. 2008;14(2):115-23. https:// doi.org/10.1089/acm.2007.7204

31. Courtney R, Greenwood KM. Preliminary investigation of a measure of dysfunctional breathing symptoms: The Self Evaluation of Breathing Questionnaire (SEBQ). Int J Osteopath Med. 2009;12(4):121-7. https://doi.org/10.1016/j.ijosm.2009.02.001

32. Agache I, Ciobanu C, Paul G, Rogozea L. Dysfunctional breathing phenotype in adults with asthma - incidence and risk factors. Clin Transl Allergy. 2012;2(1):18. https://doi.org/10.1186/2045-7022-2-18

33. Combes $P$, Fauvage B. Combined effects of hypocapnia and nicardipine on airway resistance: a pilot study. Eur J Clin Pharmacol. 1997;51(5):385-8. https://doi.org/10.1007/s002280050218

34. Brijker F, van den Elshout FJ, Heijdra YF, Bosch FH, Folgering HT. Effect of acute metabolic acid/base shifts on the human airway calibre. Respir Physiol. 2001;124(2):151-8. https://doi.org/10.1016/ S0034-5687(00)00196-1

35. Miller MR, Hankinson J, Brusasco V, Burgos F, Casaburi R, Coates A et al. Standardisation of spirometry. Eur Respir J. 2005:26(2):319-38. https://doi.org/10.1183/09031936.05.00034805

36. Courtney $R$, van Dixhoorn J, Greenwood KM, Anthonissen EL. Medically unexplained dyspnea: partly moderated by dysfunctional (thoracic dominant) breathing pattern. J Asthma. 2011;48(3):259-65. https://doi.org/10.3109/02770903.2011.554942

37. Courtney R, Cohen M, Reece J. Comparison of the Manual Assessment of Respiratory Motion (MARM) and the Hi Lo Breathing Assessment in determining a simulated breathing pattern. Int J Osteopath Med. 2009;12(3):86-91. https://doi.org/10.1016/j. ijosm.2008.10.002

38. De Troyer A, Estenne M. Functional anatomy of the respiratory muscles. Clin Chest Med. 1988;9(2):175-93.

39. Janssens L, Pijnenburg M, Claeys K, McConnell AK, Troosters $T$, Brumagne $S$. Postural strategy and back muscle oxygenation during inspiratory muscle loading. Med Sci Sports Exerc. 2013;45(7):135562. https://doi.org/10.1249/MSS.0b013e3182853d27

40. Sieck GC, Ferreira LF, Reid MB, Mantilla CB. Mechanical properties of respiratory muscles. Compr Physiol. 2013;3(4):1533-67. https:// doi.org/10.1002/cphy.c130003

41. Hruska Jr R. Influences of dysfunctional respiratory mechanics on orofacial pain. Dent Clin North Am. 1997;41(2):211-27.

42. Wolf SG. Diaphragmatic spasm : a neglected cause of dyspnoea and chest pain. Integr Physiol Behav Sci. 1994;29(1):74-6. https://doi. org/10.1007/BF02691282

43. O'Donnell DE. Hyperinflation, dyspnea, and exercise intolerance in chronic obstructive pulmonary disease. Proc Am Thorac Soc. 2006;3(2):180-4. https://doi.org/10.1513/pats.200508-093DO 
44. Lewit K. Manipulative therapy in rehabilitation of the locomotor system. 3rd ed. Oxford: Butterworth Heinemann; 1999.

45. Siafakas NM, Vermeire $P$, Pride NB, Paoletti P, Gibson J, Howard P, et al. Optimal assessment and management of chronic obstructive pulmonary disease (COPD). The European Respiratory Society Task Force. Eur Respir J. 1995;8(8):1398-420. https://doi.org/10.1183/090 31936.95.08081398

46. Global Initiative for Asthma. Global strategy for asthma management and prevention. Bethesda: Global Initiative for Asthma; 2016.

47. Cluff RA. Chronic hyperventilation and its treatment by physiotherapy: discussion paper. J R Soc Med. 1984;77(10):855-62. https://doi. org/10.1177/014107688407701011

48. Law N, Ruane LE, Low K, Hamza K, Bardin PG. Dysfunctional breathing is more frequent in chronic obstructive pulmonary disease than in asthma and in health. Respir Physiol Neurobiol. 2018;247:2023. https://doi.org/10.1016/j.resp.2017.08.011

49. Courtney R, van Dixhoorn J, Cohen M. Evaluation of breathing pattern: comparison of a Manual Assessment of Respiratory Motion (MARM) and respiratory induction plethysmography. Appl Psychophysiol Biofeedback. 2008;33(2):91-100. https://doi. org/10.1007/s10484-008-9052-3

50. American Thoracic Society/European Respiratory Society. ATS/ERS Statement on respiratory muscle testing. Am J Respir Crit Care Med. 2002;166(4):518-624. https://doi.org/10.1164/rccm.166.4.518

51. Yasuma F, Hayano J. Respiratory sinus arrhythmia: why does heartbeat synchronize with respiratory rhythm? Chest. 2004;125(2):683-90. https://doi.org/10.1378/chest.125.2.683

52. Joseph CN, Porta C, Casucci G, Casiraghi N, Maffeis M, Rossi $M$, et al. Slow breathing improves arterial baroreflex sensitivity and decreases blood pressure in essential hypertension. Hypertension. 2005;46(4):714-8. https://doi.org/10.1161/01. HYP.0000179581.68566.7d

53. Volterrani M, Scalvini S, Mazzuero G, Lanfranchi P, Colombo R, Clark $A L$, et al. Decreased heart rate variability in patients with chronic obstructive pulmonary disease. Chest. 1994;106(5):1432-7. https:// doi.org/10.1378/chest.106.5.1432

54. Suzuki M, Hiroshi T, Aoyama T, Tanaka M, Ishii H, Kisohara M, et al. Nonlinear measures of heart rate variability and mortality risk in hemodialysis patients. Clin J Am Soc Nephrol. 2012;7(9):1454-60. https://doi.org/10.2215/CJN.09430911

55. Stein PK, Nelson P, Rottman JN, Howard D, Ward SM, Kleiger RE, et al. Heart rate variability reflects severity of COPD in PiZ alpha1antitrypsin deficiency. Chest. 1998;113(2):327-33. https://doi. org/10.1378/chest.113.2.327

56. Courtney $R$, Cohen $M$, van Dixhoorn J. Relationship between dysfunctional breathing patterns and ability to achieve target heart rate variability with features of "coherence" during biofeedback.
Altern Ther Health Med. 2011;17(3):38-44

57. Lum LC. Respiratory alkalosis and hypocarbia. The role of carbon dioxide in the body economy. Chest Heart Stroke J. 1978;2(1):31-4.

58. Malmberg LP, Tamminen K, Sovijärvi AR. Orthostatic increase of respiratory gas exchange in hyperventilation syndrome. Thorax 2000;55(4):295-301. https://doi.org/10.1136/thorax.55.4.295

59. Chenivesse $C$, Similowski T, Bautin N, Fournier C, Robin S, Wallaert B, et al. Severely impaired health-related quality of life in chronic hyperventilation patients: exploratory data. Respir Med 2014;108(3):517-23. https://doi.org/10.1016/..rmed.2013.10.024

60. Gosselink R, De Vos J, van den Heuvel SP, Segers J, Decramer M Kwakkel G. Impact of inspiratory muscle training in patients with COPD: what is the evidence? Eur Respir J. 2011;37(2):416-25. https://doi.org/10.1183/09031936.00031810

61. van de Weert-van Leeuwen PB, Slieker MG, Hulzebos HJ Kruitwagen $\mathrm{CL}$, van der Ent $\mathrm{CK}$, Arets HG. Chronic infection and inflammation affect exercise capacity in cystic fibrosis. Eur Respir J. 2012;39(4):893-8. https://doi.org/10.1183/09031936.00086211

62. Tobin MJ, Perez W, Guenther SM, D'Alonzo G, Dantzker DR Breathing pattern and metabolic behavior during anticipation of exercise. J Appl Physiol (1985). 1986;60(4):1306-12. https://doi. org/10.1152/jappl.1986.60.4.1306

63. Conway AV. Breathing and feeling. In: Timmons B, Ley R, editors. Behavioral and psychological approaches to breathing disorders London: Springer; 1994. p. 243-51. https://doi.org/10.1007/978-14757-9383-3_18

64. Studer R, Danuser B, Hildebrandt H, Arial M, Gomez P Hyperventilation complaints in music performance anxiety among classical music students. J Psychosom Res. 2011;70(6):557-64 https://doi.org/10.1016/j.jpsychores.2010.11.004

65. Han JN, Stegen K, Simkens K, Cauberghs M, Schepers R, Van den Bergh $\mathrm{O}$, et al. Unsteadiness of breathing in patients with hyperventilation syndrome and anxiety disorders. Eur Respir $\mathrm{J}$. 1997;10(1):167-76. https://doi.org/10.1183/09031936.97.10010167

66. Thomas M, McKinley RK, Freeman E, Foy C, Prodger P, Price D. Breathing retraining for dysfunctional breathing in asthma: randomised controlled trial. Thorax. 2003;58(2):110-5. https://doi. org/10.1136/thorax.58.2.110

67. Ritz T, Rosenfield D, Meuret AE, Bobb C, Steptoe A. Hyperventilation symptoms are linked to a lower perceived health in asthma patients. Ann Behav Med. 2008;35(1):97-104. https://doi.org/10.1007/s12160 007-9014-7

68. Lindeboom I, van der Giessen G. Hyperventilation and therapy [Article in Dutch]. Dutch J Phys Ther. 1980;90(1):18-21.

69. Jacobson E. Progressive relaxation. 2nd ed. Oxford: Univ Chicago Press; 1938. $44 \mathrm{p}$ 